\title{
SU11274 suppresses proliferation and motility of pancreatic cancer cells
}

\author{
MINORU TOMIZAWA ${ }^{1}$, FUMINOBU SHINOZAKI ${ }^{2}$, YASUFUMI MOTOYOSHI ${ }^{3}$, \\ TAKAO SUGIYAMA $^{4}$, SHIGENORI YAMAMOTO ${ }^{5}$ and NAOKI ISHIGE ${ }^{6}$ \\ Departments of ${ }^{1}$ Gastroenterology, ${ }^{2}$ Radiology, ${ }^{3}$ Neurology, ${ }^{4}$ Rheumatology, ${ }^{5}$ Pediatrics and ${ }^{6}$ Neurosurgery, \\ National Hospital Organization, Shimoshizu Hospital, Yotsukaido, Chiba 284-0003, Japan
}

Received November 9, 2014; Accepted June 16, 2015

DOI: $10.3892 / \mathrm{ol} .2015 .3452$

\begin{abstract}
Mesenchymal-epithelial transition factor (c-Met) is associated with the proliferation and motility of cancer cells. c-Met expression has been detected in surgical pancreatic cancer specimens, and its overexpression is associated with a poor prognosis. SU11274 is a specific inhibitor of c-Met. In the present study, the cell proliferation and motility of pancreatic cancer cells treated with SU11274 was investigated. The PANC-1, MIA-Paca2, NOR-P1, PK-45H, PK-1 and PK-59 pancreatic cancer cell lines were used. The expression of c-Met and cyclin D1 was analyzed by quantitative polymerase chain reaction. In addition, a 3-(4,5-dimethylthiazol-2-yl)-5-(3-carboxymethoxyphenyl)-2-(4-sulfophenyl)-2

H-tetrazolium inner salt assay was performed to assess cell proliferation, and a scratch assay was performed to assess cell motility. c-Met expression was higher in PANC-1, PK-45H, PK-1 and PK-59 cell lines compared with that in normal pancreatic tissue. Following treatment with $30 \mu \mathrm{M}$ SU11274, the proliferation of MIA-Paca 2 and PK-45H cells was suppressed to $19.8 \pm 10.7 \%(\mathrm{P}<0.05)$ and $45.8 \pm 14.8 \%(\mathrm{P}<0.05)$ of the control level, respectively. Furthermore, cyclin D1 expression was downregulated to $43.7 \pm 17.9 \%(\mathrm{P}<0.05)$ and $53.2 \pm 18.6 \%(\mathrm{P}<0.05)$ of the control level in the MIA-Paca2 and $\mathrm{PK}-45 \mathrm{H}$ cell lines, respectively, following treatment with $30 \mu \mathrm{M}$ SU11274. In addition, cell motility was reduced to $1.0 \pm 0.3 \%$ in MIA-Paca2 $(\mathrm{P}<0.05)$ and $14.7 \pm 3.5 \%$ in PK-45H $(\mathrm{P}<0.05)$ following treatment with $30 \mu \mathrm{M}$ SU11274, compared with the motility of untreated cells. These results indicated that SU11274 suppresses the proliferation of pancreatic cancer cells via the downregulation of cyclin D1. The present study also demonstrated that cell motility was suppressed by treatment with SU11274.
\end{abstract}

Correspondence to: Dr Minoru Tomizawa, Department of Gastroenterology, National Hospital Organization, Shimoshizu Hospital, 934-5 Shikawatashi, Yotsukaido, Chiba 284-0003, Japan E-mail: nihminor-cib@umin.ac.jp

Key words: mesenchymal-epithelial transition factor, quantitative polymerase chain reaction

\section{Introduction}

Pancreatic cancer is characterized by a poor prognosis and frequent early distant metastasis (1). Early detection of pancreatic cancer remains difficult, despite advances in diagnostic imaging (2). Therefore, curative surgery is rarely possible for patients with this disease. Chemotherapy is administered to patients with advanced pancreatic cancer, and has been shown to significantly improve prognosis (3). Multiple signaling pathways are involved in pancreatic cancer (1) and thus, inhibitors of these pathways may represent potential novel therapeutic targets.

Mesenchymal-epithelial transition factor (c-Met) is a receptor for hepatocyte growth factor (HGF) (4). When HGF binds to c-Met, the signal is transmitted downstream via the phosphatidylinositol 3-kinase pathway and MAP kinase. $\mathrm{HGF} / \mathrm{c}-\mathrm{Met}$ is involved with the proliferation and motility of cancer cells (5). Pancreatic cancer cells express c-Met, and overexpression of the gene encoding c-Met is associated with a poor prognosis in this disease (6). Therefore, c-Met is hypothesized to be involved in the development of pancreatic cancer. Notably, HGF is a potent mitogen in normal human pancreatic exocrine cells via its action on c-Met (7). c-Met is involved in the self-renewal of pancreatic cancer stem cells in combination with CD44 (8). This indicates that c-Met is involved in cell proliferation and that inhibitors of c-Met may present a novel molecular target for the treatment of pancreatic cancer. A previous study demonstrated that treatment with monoclonal antibodies against c-Met, suppressed tumor growth in a mouse xenograft model of pancreatic cancer and improved the survival of the mice (9). SU11274 is a specific inhibitor of c-Met, which competes with adenosine triphosphate to bind with the activation loop of c-Met (10). Therefore, in the present study, the suppression of proliferation and motility of pancreatic cancer cells following treatment with SU11274 was investigated.

\section{Materials and methods}

Cell culture. The PANC-1, MIA-Paca2, NOR-P1, PK-45H, PK-1 and PK-59 pancreatic cancer cell lines were obtained from RIKEN Cell Bank (Tsukuba, Japan). The MIA-Paca2 cell line was cultured in Dulbecco's modified Eagle's medium (DMEM; Sigma-Aldrich, St. Louis, MO, USA) and the NOR-P1, PK-45H, PK-1 and PK-59 cell lines were cultured in RPMI-1640 medium 

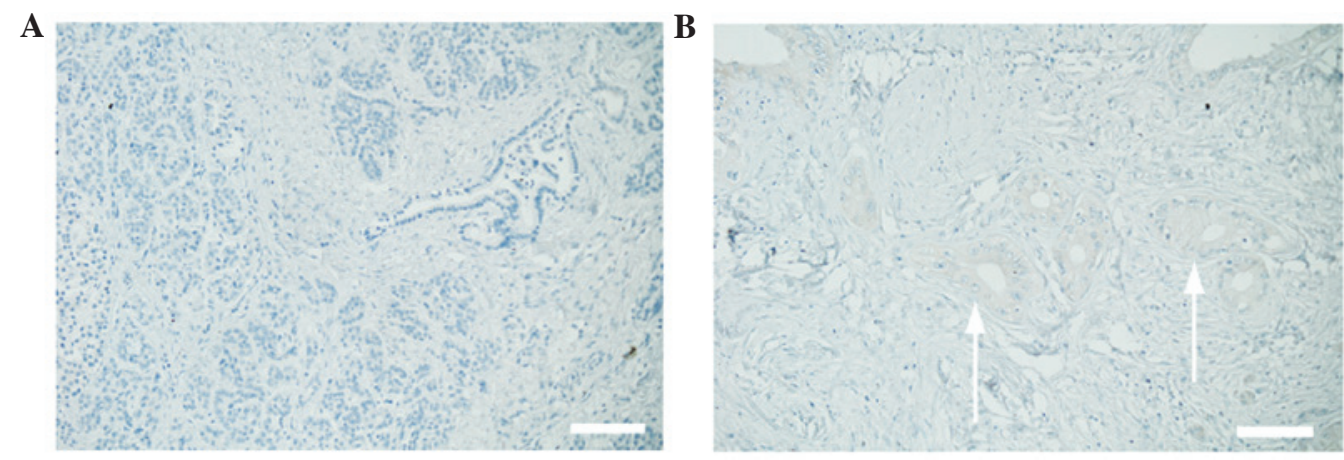

Figure 1. Immunostaining of a surgical pancreatic cancer specimen using antibodies to c-Met. (A) Normal pancreatic tissue was negative for c-Met, while (B) pancreatic cancer tissues were positive for c-Met. Arrows indicate cancer cells (magnification, x100; scale bar, 100 $\mu$ m). c-Met, mesenchymal-epithelial transition factor.

(Sigma-Aldrich), supplemented with $10 \%$ fetal bovine serum (FBS; Life Technologies, Grand Island, NY, USA). Cell lines were cultured in a humidified atmosphere of $5 \% \mathrm{CO}_{2}$ at $37^{\circ} \mathrm{C}$. The cultured cells were then observed under a microscope (CKX41N-31PHP; Olympus Corporation, Tokyo, Japan).

Scratch assay. Cells were grown on 4-well chamber slides (Becton-Dickinson, Franklin Lakes, NJ, USA). When the cells had reached $80 \%$ confluence, cell sheets were scratched with a sterile razor and 0 or $30 \mu \mathrm{M}$ SU11274 (Wako Pure Chemical Industries, Ltd., Tokyo, Japan) was added to the media. Two days later, cells were stained with hematoxylin and eosin (Muto Pure Chemicals Co., Ltd., Tokyo, Japan). The cells were then observed and images were captured using an AX80 microscope (Olympus Corporation). The number of cells that had migrated $>150 \mu \mathrm{m}$ from the cut surface were counted in 5 different fields from each slide.

Immunostaining. Serial sections of human pancreatic tissue from a 62-year-old male with adenocarcinoma and normal human pancreatic tissue from a 74-year-old male were purchased from BioChain (Hayward, CA, USA). The sections were deparaffinized, autoclaved and incubated with hydrogen peroxide, followed by $2 \%$ FBS in phosphate-buffered saline (PBS) for $30 \mathrm{~min}$. After incubation overnight at $4^{\circ} \mathrm{C}$ with a monoclonal rabbit anti-human c-Met antibody (cat. no. 8198; 1:300; Cell Signaling Technology, Danvers, MA, USA), specimens were washed with PBS and subsequently incubated with a polyclonal horseradish-peroxidase-labeled donkey anti-rabbit secondary antibody (cat. no. NA934-100UL; 1:2,000; GE Healthcare, Pittsburgh, PA, USA) for $2 \mathrm{~h}$ at $4^{\circ} \mathrm{C}$. Subsequently, diaminobenzidine (Dako, Glostrup, Denmark) was applied as the chromogen to tissue sections and nuclei were stained with hematoxylin for $15 \mathrm{sec}$. Specimens were observed and images were captured under the AX80 microscope.

Cell proliferation assay. MIA-Paca2 and PK-45H cells were trypsinized, harvested and seeded in 96-well flat-bottom plates (Asahi Glass Co., Ltd., Tokyo, Japan) at a density of 1,000 cells/well. MIA-Paca2 and PK-45H cells were incubated for $24 \mathrm{~h}$ in DMEM and RPMI-1640 medium, respectively, supplemented with $10 \%$ FBS. Following cell culture, the c-Met inhibitor, SU11274, was added at the desired concentrations $(0$ or $30 \mu \mathrm{M})$ and cells were incubated for $72 \mathrm{~h}$. Cell proliferation was then assessed by a 3-(4,5-dimethylthiazol-2-yl)-5-(3-carboxymethoxyphenyl)-2-(4-sulfophenyl)-

2H-tetrazolium (MTS), inner salt assay, according to the manufacturer's instructions (Promega Corporation, Madison, WI, USA). MTS is reduced by cells to a colored formazan product, with a maximum absorbance at $490 \mathrm{~nm}$. Absorbance at $490 \mathrm{~nm}$ was measured with an iMark Microplate Absorbance Reader (Bio-Rad, Hercules, CA, USA).

Quantitative polymerase chain reaction ( $q P C R)$. Cells were cultured in 6-well plates (Asahi Glass Co., Ltd.). Forty-eight hours after the addition of SU11274 to the media, total RNA was isolated with Isogen reagent (Nippon Gene, Tokyo, Japan). Complementary DNA was synthesized with SuperScript III and oligo(dT) primers according to the manufacturer's instructions (Life Technologies). Normal pancreatic tissue total RNA was purchased from Clontech Laboratories, Inc., (Mountain View, CA, USA). The following PCR primers were used: Forward, 5'-CATTGGGGAGCACTATGTC-3' and reverse, 5'-TGTCCA CCTCATCATCAGCG-3' for c-Met (accession no.NM_000245), $110 \mathrm{bp}$; forward, 5'-AGAGGCGGAGGAGAACAAACAG-3' and reverse, 5'-AGGCGGTAGTAGGACAGGAAGTTG-3' for cyclin D1 (accession no. NM_053056), 180 bp; and forward, 5'-CGAATGCCAGAGAAGGTCAC-3' and reverse, 5'-CCA TGAGAATCCGCTTGT TT-3' for RPL19 (accession no. BC095445), 157 bp. RPL19 was used as an internal control to monitor the amount of mRNA. Quantitative PCR was performed using Fast SYBR-Green Master Mix (Life Technologies) in a Mini Opticon system (Bio-Rad); 40 cycles consisting of $5 \mathrm{sec}$ of denaturation at $95^{\circ} \mathrm{C}$ and $5 \mathrm{sec}$ of annealing-extension at $60^{\circ} \mathrm{C}$ were performed. The expression levels of genes were analyzed automatically by this system. The relative c-MET expression levels were calculated as follows: Relative c-Met expression $=\mathrm{c}-$ Met expression/RPL19 expression.

Statistical analysis. One-way analysis of variance was performed using JMP 10.0.2 statistical software (SAS Institute, Cary, NC, USA). $\mathrm{P}<0.05$ was considered to indicate a statistically significant difference.

\section{Results}

c-Met expression is higher in pancreatic cancer cells than in normal pancreatic cells. A commercially available surgical 


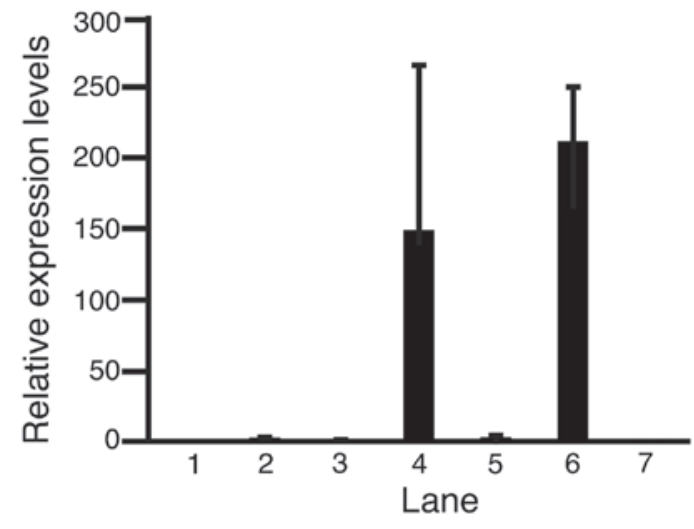

Figure 2. Quantitative polymerase chain reaction was performed to analyze expression levels of c-Met in pancreatic cancer cell lines. The expression levels were normalized against the level in normal pancreatic tissue. Lanes: 1, MIA-Paca2; 2, PANC-1; 3, NOR-P1; 4, PK-45H; 5, PK-1; 6, PK-59H; 7, human adult pancreas. Error bar, standard deviation; $n=3$. c-Met, mesenchymal-epithelial transition factor.

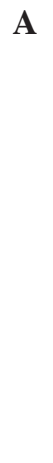

B
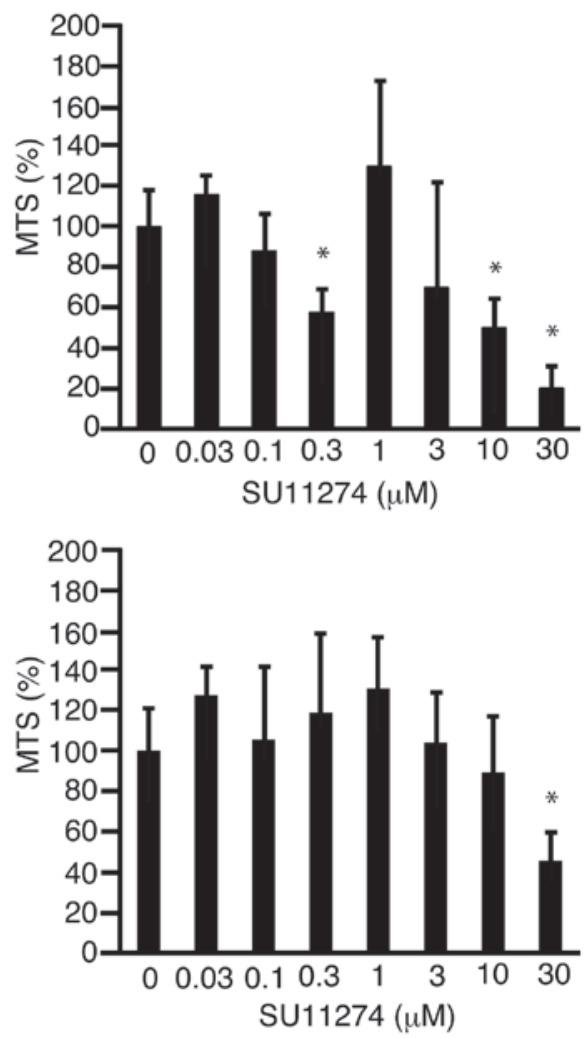

Figure 3. MTS inner salt cell proliferation assays were performed with (A) MIA-Paca2 and (B) PK-45H cells at $72 \mathrm{~h}$ following treatment with various concentrations of SU11274. ${ }^{*} \mathrm{P}<0.05$ vs. $0 \mu \mathrm{M}$ SU11274; $\mathrm{n}=3$. Data are expressed as the mean \pm standard deviation.

specimen was immunostained in order to determine whether pancreatic cancer cells express c-Met. Normal pancreatic tissue was negative for c-Met (Fig. 1A), while the cell surface and cytoplasm of pancreatic cancer cells were positive for c-Met (Fig. 1B). Therefore, the results demonstrated that c-Met is only expressed in pancreatic cancer cells.

Expression levels of c-Met in normal pancreatic cells and pancreatic cancer cell lines were compared using quantitative PCR, and normalized against that of the normal
A

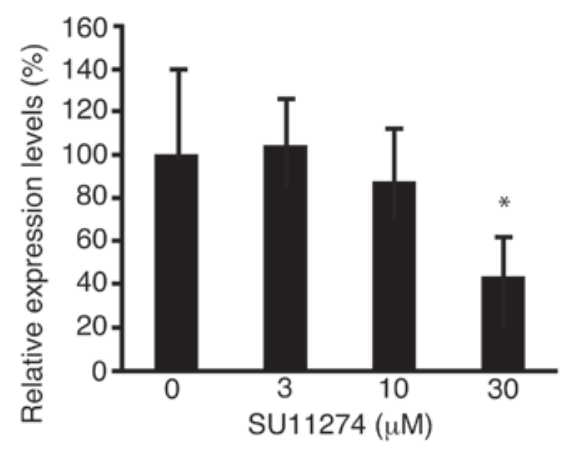

B

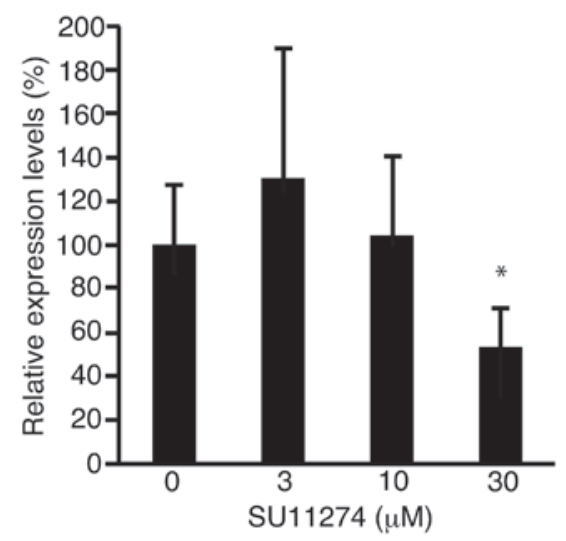

Figure 4. Quantitative polymerase chain reaction was performed to analyze the expression level of cyclin D1 in (A) MIA-Paca2 and (B) PK-45H cell lines. Data are expressed as the mean \pm standard deviation. ${ }^{*} \mathrm{P}<0.05$ vs. $0 \mu \mathrm{M}$ SU11274; $\mathrm{n}=3$.

pancreas (Fig. 2). The relative expression levels of c-Met in the MIA-Paca2, PANC-1, NOR-P1, PK-45H, PK-1 and PK-59 cell lines and normal pancreatic cells were $0.05 \pm 0.01$, $2.66 \pm 0.15,0.98 \pm 0.60,1.49 \pm 1.16,2.92 \pm 2.00,2.12 \pm 0.38$ and $1.00 \pm 0.20$ (mean \pm standard deviation), respectively. c-Met expression was higher in PANC-1, PK-45H, PK-1 and PK-59 cell lines than that in normal pancreatic cells. These results indicate that $\mathrm{c}-\mathrm{Met}$ is involved in the development of pancreatic cancer. The MIA-Paca2 and PK-45H cell lines were selected for further analysis; PK-45H cells exhibited increased c-Met expression compared with that in normal pancreatic cells, while MIA-Paca2 cells exhibited reduced c-Met expression compared with that normal pancreatic cells.

SU11274 suppresses pancreatic cancer cell proliferation. SU11274 was added to the culture media and an MTS assay was performed in order to determine whether this inhibitor of c-Met suppressed cell proliferation. Proliferation of MIA-Paca2 and PK-45H cells was suppressed to $19.8 \pm 10.7 \%$ ( $\mathrm{P}<0.05$; Fig. 3A) and $45.8 \pm 14.8 \%(\mathrm{P}<0.05$; Fig. $3 \mathrm{~B})$ of the control level, respectively, following treatment with $30 \mu \mathrm{M}$ SU11274.

Cyclin D1 is involved in cell cycle progression. Expression of the cyclin D1 gene was measured by quantitative PCR. In the MIA-Paca2 and PK-45H cell lines, cyclin D1 expression was downregulated to $43.7 \pm 17.9 \%(\mathrm{P}<0.05$; Fig. $4 \mathrm{~A})$ and $53.2 \pm 18.6 \%(\mathrm{P}<0.05$; Fig. 4B) of the control level, respectively, following treatment with $30 \mu \mathrm{M}$ SU11274.

SU11274 suppresses pancreatic cell motility. A scratch assay was performed in order to assess the inhibition of cell motility 

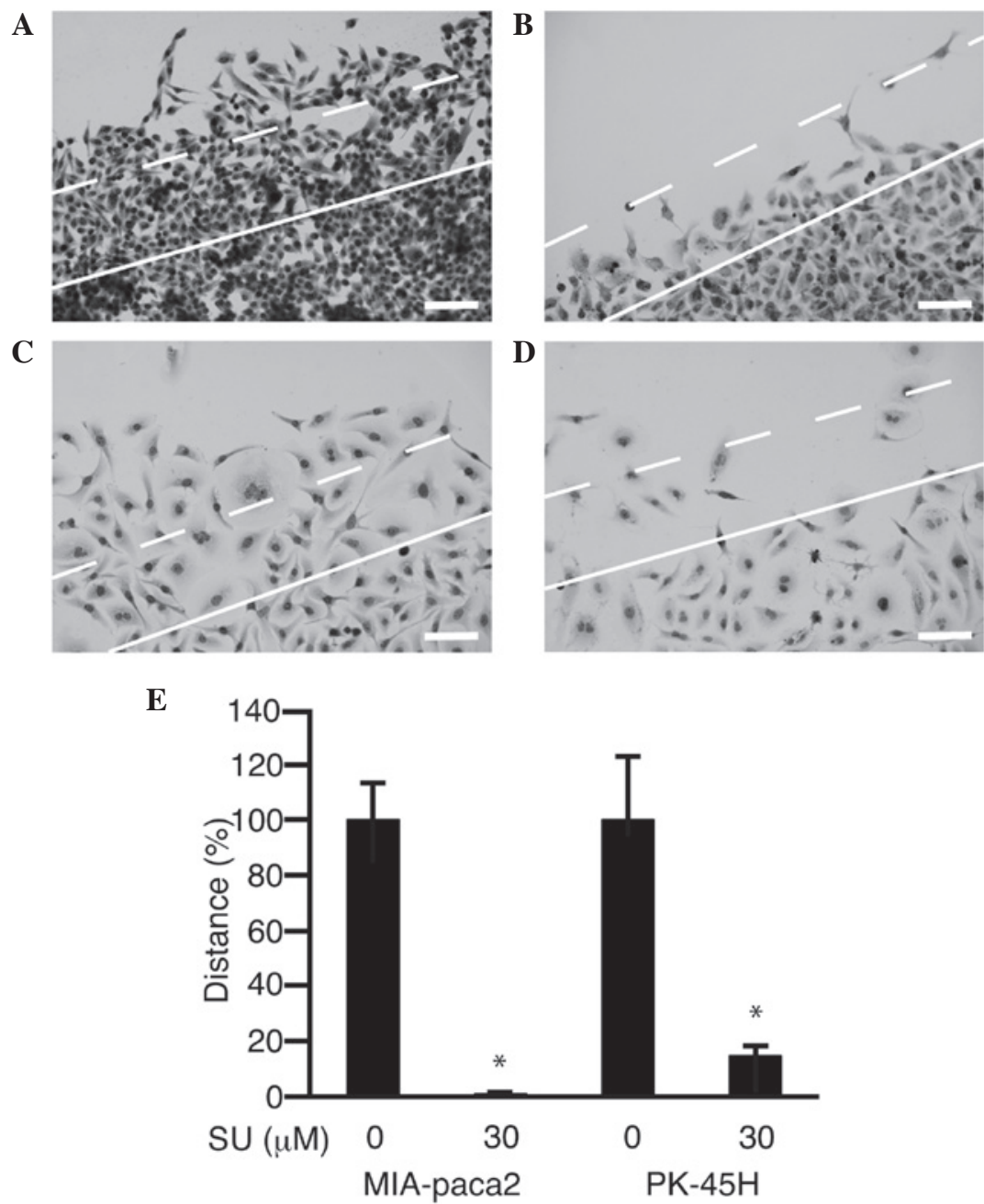

Figure 5. Scratch assay. MIA-Paca2 cells and PK-45H cells were cultured in 4-well chamber slides and scratched with a sterile razor. After $48 \mathrm{~h}$ of culture with or without SU11274 $(30 \mu \mathrm{M})$, images were captured. The number of cells that had migrated $>150 \mu \mathrm{m}$ from the cut surface were counted. (A) Untreated MIAPaca2 cells. (B) MIA-Paca2 treated with $30 \mu \mathrm{M}$ SU11274. (C) Untreated PK-45H cells. (D) PK-45H cells treated with $30 \mu$ M SU11274. Magnification, x200; scale bar, $100 \mu \mathrm{m}$. Solid line, cut surface; broken line, $150 \mu \mathrm{m}$ marker from the cut surface. (E) The cell numbers in SU11274-treated cultures were normalized against numbers in untreated cultures. Data are expressed as the mean \pm standard deviation. ${ }^{*} \mathrm{P}<0.05$ vs. $0 \mu \mathrm{M}$ SU11274; $\mathrm{n}=3$. SU, SU11274.

following treatment with SU11274. Forty-eight hours after scratching of the cell sheet, untreated cells had migrated $>150 \mu \mathrm{m}$ from the cut surface (Fig. 5A and C). In cell cultures treated with SU11274 $(30 \mu \mathrm{M})$, a lower number of cells had migrated (Fig. 5B and D). Cells that had moved $>150 \mu \mathrm{m}$ were counted (Fig. 5E). Following treatment with $30 \mu \mathrm{M}$ SU11274, cell motility was suppressed to $1.0 \pm 0.3 \%$ in MIA-Paca2 cells $(\mathrm{P}<0.05)$ and $14.7 \pm 3.5 \%$ in $\mathrm{PK}-45 \mathrm{H}$ cells $(\mathrm{P}<0.05)$ of the level of cells treated with $0 \mu \mathrm{M}$ SU11274, respectively.

\section{Discussion}

c-Met expression has been identified in pancreatic cancer specimens (11). In the present study, immunostaining detected no c-Met expression at the protein level in normal pancreatic tissues. These results indicated that c-Met is involved in the development of pancreatic cancer. HGF is a potent mitogen in normal human pancreatic exocrine tissue and functions in an autocrine manner $(7,8)$. Thus, it was hypothesized that an inhibitor of c-Met may suppress the proliferation of pancreatic cancer cells. SU11274 is a specific inhibitor of c-Met, which has been shown to suppress the proliferation of hepatocellular carcinoma cells (12). In the present study, SU11274 effectively suppressed the proliferation of pancreatic cancer cells via downregulation of cyclin D1.

c-Met is involved in cell motility and tumor-stromal interactions (13) and thus, inhibition of c-Met suppresses cell motility (14). In the present study, SU11274 significantly suppressed the motility of pancreatic cancer cells. Cancer cells migrate and invade the surrounding tissues in clusters (15). Pancreatic tumors characteristically exhibit abundant stroma, and this microenvironment represents a novel therapeutic target (16). c-Met inhibitors, such as SU11274, may therefore be developed for use in the treatment of pancreatic cancer.

Quantitative PCR demonstrated that c-Met was not expressed in all pancreatic cancer cell lines. The expression of the c-Met gene was lower in the MIA-Paca2 cell line compared with that in normal pancreatic cells. However, SU11274 nevertheless suppressed proliferation in this cell line. Although the mechanism remains unclear, SU11274 may exert 
antitumor effects in all pancreatic cancer cell lines, regardless of the level of c-Met expression.

At present, c-Met inhibitors, such as tivantinib, are undergoing clinical trials in patients with pancreatic cancer (17). Combined treatment with tivantinib and gemcitabine is safe and tolerable (17). Although these are preliminary results, the antitumor effect of this combination appears promising. Crizotinib, another c-Met inhibitor, inhibits the inactivation of gemcitabine by pancreatic cancer cells (18). These studies indicate that combining c-Met inhibitors and gemcitabine may present a novel strategy for the treatment of pancreatic cancer.

The insulin-like growth factor 1 receptor (IGF-1R) is involved in pancreatic cancer cell proliferation, and its inhibition suppresses cell proliferation and motility $(19,20)$. Notably, combined treatment with a c-Met inhibitor and IGF-1R inhibitor has been shown to suppress the proliferation of pancreatic cancer cells in vitro and in vivo (21). Thus, future studies investigating combined treatment with inhibitors of c-Met, IGF-1R and the Wnt pathway are required (22).

In conclusion, in the current study SU11274 suppressed the proliferation of pancreatic cancer cells via the downregulation of cyclin D1. Cell motility was also suppressed following treatment with SU11274. Thus, c-Met may present a promising therapeutic target for pancreatic cancer.

\section{References}

1. Wörmann SM and Algül H: Risk factors and therapeutic targets in pancreatic cancer. Front Oncol 3: 282, 2013.

2. Lennon AM, Wolfgang CL, Canto MI, Klein AP, Herman JM, Goggins M, Fishman EK, Kamel I, Weiss MJ, Diaz LA, et al: The early detection of pancreatic cancer: What will it take to diagnose and treat curable pancreatic neoplasia? Cancer Res 74: 3381-3389, 2014.

3. Gresham GK, Wells GA, Gill S, Cameron C and Jonker DJ: Chemotherapy regimens for advanced pancreatic cancer: A systematic review and network meta-analysis. BMC Cancer 14 471, 2014.

4. Organ SL and Tsao MS: An overview of the c-MET signaling pathway. Ther Adv Med Oncol 3 (Suppl): S7-S19, 2011.

5. Goetsch L, Caussanel V and Corvaia N: Biological significance and targeting of c-Met tyrosine kinase receptor in cancer. Front Biosci (Landmark Ed) 18: 454-473, 2013.

6. Zhu GH, Huang C, Qiu ZJ, Liu J, Zhang ZH, Zhao N, Feng ZZ and Lv XH: Expression and prognostic significance of CD151, c-Met, and integrin alpha3/alpha6 in pancreatic ductal adenocarcinoma. Dig Dis Sci 56: 1090-1098, 2011.

7. Vilá MR, Nakamura T and Real FX: Hepatocyte growth factor is a potent mitogen for normal human pancreas cells in vitro. Lab Invest 73: 409-418, 1995.
8. Li C, Wu JJ, Hynes M, Dosch J, Sarkar B, Welling TH, Pasca di Magliano M and Simeone DM: c-Met is a marker of pancreatic cancer stem cells and therapeutic target. Gastroenterology 141: 2218-2227, 2011.

9. Jin H, Yang R, Zheng Z, Romero M, Ross J, Bou-Reslan H, Carano RA, Kasman I, Mai E, Young J, et al: MetMAb, the one-armed 5D5 anti-c-Met antibody, inhibits orthotopic pancreatic tumor growth and improves survival. Cancer Res 68: 4360-4368, 2008.

10. Mughal A, Aslam HM, Sheikh A, Khan AM and Saleem S: c-Met inhibitors. Infect Agent Cancer 8: 13, 2013.

11. Gardian K, Janczewska S and Durlik M: Microenvironment elements involved in the development of pancreatic cancer tumor. Gastroenterol Res Pract 2012: 585674, 2012.

12. Inagaki Y, Qi F, Gao J, Qu X, Hasegawa K, Sugawara Y, Tang W and Kokudo N: Effect of c-Met inhibitor SU11274 on hepatocellular carcinoma cell growth. Biosci Trends 5: 52-56, 2011.

13. Yasui T, Ohuchida K, Zhao M, Onimaru M, Egami T, Fujita H, Ohtsuka T, Mizumoto K and Tanaka M: Tumor-stroma interactions reduce the efficacy of adenoviral therapy through the HGF-MET pathway. Cancer Sci 102: 484-491, 2011.

14. Chan B, VanderLaan PA and Sukhatme VP: 6-Phosphogluconate dehydrogenase regulates tumor cell migration in vitro by regulating receptor tyrosine kinase c-Met. Biochem Biophys Res Commun 439: 247-251, 2013

15. Tomizawa M, Kondo F and Kondo Y: Growth patterns and interstitial invasion of small hepatocellular carcinoma. Pathol Int 45: 352-358, 1995.

16. Neesse A, Krug S, Gress TM, Tuveson DA and Michl P: Emerging concepts in pancreatic cancer medicine: Targeting the tumor stroma. Onco Targets Ther 7: 33-43, 2013.

17. Pant S, Saleh M, Bendell J, Infante JR, Jones S, Kurkjian CD, Moore KM, Kazakin J, Abbadessa G, Wang Y, et al: A phase I dose escalation study of oral c-MET inhibitor tivantinib (ARQ 197) in combination with gemcitabine in patients with solid tumors. Ann Oncol 25: 1416-1421, 2014.

18. Avan A, Caretti V, Funel N, Galvani E, Maftouh M, Honeywell RJ, Lagerweij T, Van Tellingen O, Campani D, Fuchs D, et al: Crizotinib inhibits metabolic inactivation of gemcitabine in c-Met-driven pancreatic carcinoma. Cancer Res 73: 6745-6756, 2013.

19. Tomizawa M, Shinozaki F, Sugiyama T, Yamamoto S, Sueishi $\mathrm{M}$ and Yoshida T: Insulin-like growth factor-I receptor in proliferation and motility of pancreatic cancer. World $\mathrm{J}$ Gastroenterol 16: 1854-1858, 2010.

20. Tomizawa M, Shinozaki F, Sugiyama T, Yamamoto S, Sueishi M and Yoshida T: Insulin-like growth factor I receptor involvement in proliferation of NOR-P1 cells in serum-free media. J Cell Biochem 113: 2714-2720, 2012.

21. Ucar DA, Magis AT, He DH, Lawrence NJ, Sebti SM, Kurenova E, Zajac-Kaye M, Zhang J and Hochwald SN: Inhibiting the interaction of cMET and IGF-1R with FAK effectively reduces growth of pancreatic cancer cells in vitro and in vivo. Anticancer Agents Med Chem 13: 595-602, 2013.

22. Tomizawa M, Shinozaki F, Sugiyama T, Yamamoto S, Sueishi M and Yoshida T: Frizzled-2: A potential novel target for molecular pancreatic cancer therapy. Oncol Lett 7: 74-78, 2014. 